\title{
MINIMALLY THIN SETS BELOW A FUNCTION GRAPH
}

\author{
TORBJÖRN LUNDH
}

\begin{abstract}
A set $E$ is minimally thin at a boundary point, $\xi$, if the Martin kernel with pole at $\xi$ does not coincide with its balayage on $E$. Or in a probabilistic language: There is a non-zero probability that a Brownian motion that is conditioned to exit at $\xi$ will avoid the set $E$. We will consider a special class of sets $E$, namely sets in the upper half-space that lies between the graph of a function and the boundary of the half-space. Brelot and Doob gave in 1963 an integral criterion for positive non-decreasing functions for minimally thinness of $E$. In 1991 Gardiner showed that the same criterion holds for the class of Lipschitz continuous functions. We will generalize these results to the class self-controlled functions, which is similar to the Beurling slow varying class of functions.
\end{abstract}

Keywords: Self-controlled, Beurling slow varying, minimal thinness, Whitney decomposition.

2000 Mathematics Subject Classification. 30C85, 31A15, 31B05, 31B15, 31C35;

\section{INTRODUCTION}

The history of the following result is as long as the history of minimal thinness itself. The reason is probably because it deals with one of the most straightforward constructions of sets that candidate for being minimally thin at a given point. From now on we will for simplicity assume that the given point is the origin.

Let us consider the upper-half-space

$$
\mathbb{H}=\left\{(x, y) \in \mathbb{R}^{\mathrm{d}}: x=\left(x_{1}, x_{2}, \ldots, x_{\mathrm{d}-1}\right) \in \mathbb{R}^{\mathrm{d}-1} \text { and } y \geq 0\right\}
$$

and let us define the subset

$$
F_{f}=\left\{(x, y) \in \mathbb{R}^{\mathrm{d}}: 0 \leq y \leq f(|X|)\right\},
$$

where $f$ is a function from $\mathbb{R}_{+}$to $[0, \infty)$.

Let us now state a well known result, originally stated in the upper half plane by M. Brelot and J. Doob in 1963.

Theorem A. ([6], see also [1, p. 440], [7, p. 98], [3, p. 357]) Let $f$ be a positive non-decreasing function on $(0, \infty)$. Then

$$
F_{f} \text { is minimally thin at } 0 \text { if and only if } \int_{0} \frac{f(x)}{x^{2}} d x<\infty \text {. }
$$

This paper is dedicated in memory of my teacher Matts Essén. He was the most caring teacher you could which for. I am most grateful to have been one of his students and friends. In the present study, I was originally inspired by a Wiener-type criterion for minimal thinness (using a Whitney decomposition) which was introduced by Matts Essén in [9]. 
K. Burdzy gave a probabilistic proof in [7, Theorem 8.2 and Remark 8.2] where he also raised the question how to extend to functions not being non-decreasing.

Using Lipschitz continuous functions, S. Gardiner gave in the following result a way to extend Theorem A to functions $f$ not necessarily non-decreasing. Using his formulation we will look at sets that are not necessarily radially symmetric, i.e let

$$
E_{\varphi}=\left\{(x, y) \in \mathbb{R}^{\mathrm{d}}: x \in \mathbb{R}^{\mathrm{d}-1}, \text { and } 0 \leq y \leq \varphi(x)\right\},
$$

where $\varphi$ is a function from $\mathbb{R}^{\mathrm{d}-1}$ to $[0, \infty)$ and such that $\varphi(0)=0$.

Theorem B. ([10, Lemma 1]) Let $E_{\varphi}$ be as in (2) and such that $\varphi$ is such that

$$
|\varphi(x)-\varphi(y)| \leq a|x-y| \text { for } x, y \in \mathbb{R}^{d-1} \text {. }
$$

Then $E_{\varphi}$ is minimally thin at 0 if and only if

$$
\int_{0} \frac{\varphi(x)}{|x|^{d}} d x<\infty
$$

\section{Self Controlled functions}

We will now use a more general condition on $\varphi$, based on the definition of selfcontrolled functions introduced by C. Goldie and R. Smith in [11], which we here formulate near the origin instead of a neighborhood of infinity.

Definition 2.1. ( $[11,(2.4 .2)]$ ) If $f$ is a measurable function from $(0, \infty)$ to $(0, \infty)$. We say that $f$ is self-controlled at 0 if for some $\Delta, c_{s}, C>0, x_{0} \geq 0$,

$$
c_{s} f(x) \leq f(x+\delta f(x)) \leq C f(x) \text { for some } 0 \leq \delta \leq \Delta, x \leq x_{0} .
$$

We will use a generalization of their definition in the following one sided, higher dimensional, variant.

Definition 2.2. If $\varphi$ is a positive function on $\mathbb{R}^{d-1}$. We say that $\varphi$ is lower self-controlled at 0 if it is measurable and

$$
\inf _{\substack{|v| \leq \Delta \varphi(x) \\ \operatorname{sign}\left(v_{i}\right) \operatorname{sign}\left(x_{i}\right) \geq 0}} \frac{\varphi(x+v)}{\varphi(x)}=c_{s}>0 \text { for some } \Delta>0 .
$$

Remark 2.3. Heuristically, a lower self-controlled function can not decrease too fast, but is allowed to increase momentarily, when "going out" away from the origin. See Figure 2 for a one-dimensional example of a lower self-controlled function (which is not self-controlled).

Remark 2.4. It should be noted that the (lower) self-controlled condition is a close relative to the so called Beurling slow varying condition, see for example [5, p. 120]. That is worth noting, especially since also the other concept of interest in this short paper, minimal thinness, is indicated in the seminal paper [4] of A. Beurling.

Let us now formulate our main result.

Theorem 2.5. Let $\varphi$ be lower self-controlled, then $E_{\varphi}$ is minimally thin in the upper half space at the origin if and only if (4) holds 
3. Proof of Theorem 2.5

Definition 3.1. We say that $\left\{Q_{k}\right\}$ is a Whitney decomposition in the upperhalf-space $\mathbb{H}$ if $\left\{Q_{k}\right\}$ is a partition of $\mathbb{H}$ and if all $Q_{k}$ are cubes (or squares) in $\mathbb{H}$ and there exists universal constants $c^{\prime}$ and $c$ such that

$$
c^{\prime} t_{k} \leq \text { sidelength of } Q_{k} \leq c t_{k},
$$

where $t_{k}$ is the distance from the center of $Q_{k}$ to the boundary $\partial \mathbb{H}$.

We will also need the corresponding two Whitney adjusted variants of our set $E_{\varphi}$

$$
\begin{aligned}
& \overline{E_{\varphi}}=\bigcup_{Q_{k} \cap E_{\varphi} \neq \emptyset} Q_{k}, \\
& \underline{E_{\varphi}}=\bigcup_{Q_{k} \cap E_{\varphi}=Q_{k}} Q_{k} .
\end{aligned}
$$

Definition 3.2. Let us by $\mathcal{M}$ denote the class of functions from $\mathbb{R}^{d-1}$ to $\mathbb{R}$ such that for each $\varphi$ in $\mathcal{M}$ there exists a Whitney decomposition $\left\{Q_{k}\right\}$ in $\mathbb{H}^{d-1}$ such that

$$
\int_{\underline{E_{\varphi}}} \frac{d z}{|z|^{d}}<\infty \Rightarrow \int_{\overline{E_{\varphi}}} \frac{d z}{|z|^{d}}<\infty .
$$

We will need the following theorem of B. Dahlberg, which we state in the form given in $[2$, p. 26].

Theorem C. ([8, Theorem 1]) $\overline{E_{\varphi}}$ is minimally thin at 0 if and only if

$$
\int_{\overline{E_{\varphi}}} \frac{d z}{|z|^{d}}<\infty
$$

Let us also define two auxiliary functions that approximates $\varphi$. The upper Whitney function of $\varphi$ is defined as

$$
\bar{\varphi}(x)=\sup \left\{y:(x, y) \in Q_{k}, \text { such that } Q_{k} \cap(x, \varphi(x)) \neq \emptyset\right\},
$$

and the lower Whitney function of $\varphi$ is defined as

$$
\underline{\varphi}(x)=\inf \left\{y:(x, y) \in Q_{k}, \text { such that } Q_{k} \cap(x, \varphi(x)) \neq \emptyset\right\},
$$

where $x \in R^{\mathrm{d}-1}$.

Note that $E_{\bar{\varphi}}=\overline{E_{\varphi}}$ and $E_{\underline{\varphi}}=\underline{E_{\varphi}}$.

Lemma 3.3. If $\varphi \in \mathcal{M}$ then $E_{f}$ is minimally thin at 0 if and only if (4) holds.

Proof. We note that

$$
\underline{E_{\varphi}} \subset E_{\varphi} \subset \overline{E_{\varphi}}
$$

and thus, if $\varphi \in \mathcal{M}$ then, thanks to Theorem $\mathrm{C}$, we see that $E_{\varphi}$ is minimally thin if and only if $\int_{E_{\varphi}} \frac{d z}{|z|^{\mathrm{d}}}<\infty$, i.e. (4).

Proof of Theorem 2.5. From Lemma 3.3 we see that it is sufficient to show that $\varphi$ is lower self-controlled implies that $\varphi \in \mathcal{M}$. Let us therefore suppose that $\varphi$ is self-controlled with the constants $\Delta$ and $c_{s}$ as in Definition 2.2 above.

Let us now pick a Whitney decomposition of $\mathbb{H}$ such that the conditions in Definition 3.1 are fulfilled with the constant $c \leq \min \left(\frac{2 c_{s}}{4+c_{s}}, \frac{2 \Delta}{2 \sqrt{\mathrm{d}-1}+\Delta}\right)$. 


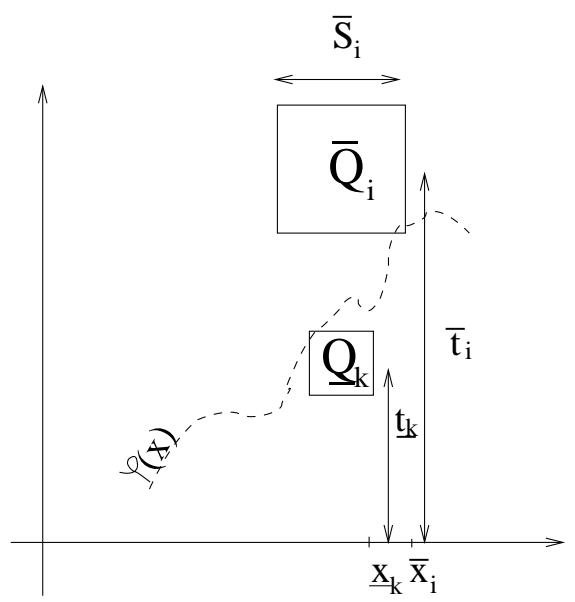

Figure 1. An upper and a lower Whitney square.

Let us by $s_{k}$ denote the side-length of the cube $Q_{k}$. Definition 3.1 give us then that

$$
c^{\prime} t_{k} \leq s_{k} \leq c t_{k}
$$

We will study those Whitney cubes whose lower edge coincides with $\underline{\varphi}$ and denote them $\left\{\underline{Q}_{k}\right\}$. We will also use the notion $\underline{t}$ for the distance from the center of the cube $\underline{Q}_{k}$ to the boundary $\partial \mathbb{H}$, and $\underline{s}$ for the side length of such lower cubes.

Completely analogously we have similar notions for the squares $\left\{\bar{Q}_{k}\right\}$ connected to $\bar{\varphi}$.

Let $b\left(Q_{k}\right)$ be the projection of of the Whitney cube $Q_{k}$ down to $\partial \mathbb{H}$.

Let us now obtain some estimates for the squares $\left\{\underline{Q}_{k}\right\}$ below an "upper" square $\left\{\bar{Q}_{i}\right\}$, i.e. the indexes $k$ and $i$ are such that $b\left(\underline{Q}_{k}\right) \cap \bar{b}\left(\bar{Q}_{i}\right) \neq \emptyset$, e.g. see Figure 1 .

Let

$$
\operatorname{Var}_{k}=\sup _{x, \xi \in b\left(Q_{k}\right)} \varphi(x)-\varphi(\xi) .
$$

We can find a $v \in \mathbb{R}^{\mathrm{d}-1}$ such that $\xi=x+v$ and $|v| \leq \Delta \varphi(x)$, since $|v| \leq$ $\sqrt{\mathrm{d}-1} s_{k} \leq \sqrt{\mathrm{d}-1} c\left(\varphi(x)+\frac{1}{2} s_{k}\right)$, and $c<\frac{2 \Delta}{2 \sqrt{\mathrm{d}-1}+\Delta}$. Thus we have that

$$
\operatorname{Var}_{k} \leq \sup _{x \in b\left(Q_{k}\right)} \varphi(x)\left(1-c_{s}\right)
$$

For $\bar{Q}_{i}$ and $\underline{Q}_{k}$ as above, we have the following.

$$
\bar{t}_{i}-\underline{t}_{k} \leq \frac{1}{2}\left(\underline{s}_{k}+\bar{s}_{i}\right)+\operatorname{Var}_{i} \leq \bar{s}_{i}+\left(\bar{t}_{i}+\frac{1}{2} \bar{s}_{i}\right)\left(1-c_{s}\right) \leq \bar{t}_{i}\left(1+\frac{3 c}{2}-c_{s}\left(1-\frac{c}{2}\right)\right) .
$$

Let now $M=c_{s}\left(1-\frac{c}{2}\right)-\frac{3 c}{2}$. We then note that $M$ is strictly positive for our Whitney decomposition since $c \leq \frac{2 c_{s}}{4+c_{s}}$. Hence

$$
\underline{t}_{k} \geq M \bar{t}_{i} \text {. }
$$

Let us suppose that $\int_{\underline{E_{\varphi}}} \frac{d z}{|z|^{\mathrm{d}}}<\infty$ holds. Then, by summing over all $\left\{\underline{Q}_{k}\right\}$ we have that

$$
\infty>\int_{0} \frac{\underline{\varphi}(x)}{\left.|| x\right|^{\mathrm{d}}} d x \geq \sum_{\left\{\underline{Q}_{k}\right\}}\left(\underline{t}_{k}-\frac{1}{2} \underline{s}_{k}\right) \int_{b\left(\underline{Q}_{k}\right)} \frac{1}{|x|^{\mathrm{d}}} d x \geq \sum_{\left\{\underline{Q}_{k}\right\}} \underline{t}_{k}\left(1-\frac{c}{2}\right) \int_{b\left(\underline{Q}_{k}\right)} \frac{1}{|x|^{\mathrm{d}}} d x
$$


from (5). From (7) we then obtain

$$
\begin{gathered}
\infty>M\left(1-\frac{c}{2}\right) \sum_{\left\{\underline{Q}_{k}\right\}} \bar{t}_{i} \int_{b\left(\underline{Q}_{k}\right)} \frac{1}{|x|^{\mathrm{d}}} d x \geq M\left(1-\frac{c}{2}\right) \sum_{\left\{\bar{Q}_{i}\right\}} \bar{t}_{i} \int_{b\left(\bar{Q}_{i}\right)} \frac{1}{|x|^{\mathrm{d}}} d x \geq \\
\geq M \frac{\left(1-\frac{c}{2}\right)}{\left(1+\frac{c}{2}\right)} \int_{0} \frac{\bar{\varphi}(x)}{|x|^{\mathrm{d}}} d x .
\end{gathered}
$$

We conclude that $f$ is in the family $\mathcal{M}$ finishing the proof.

Is Theorem 2.5 really a generalization of the earlier results? The following lemma tells us that that is the case.

Lemma 3.4. A function $\varphi$ from $\mathbb{R}^{d-1}$ to $\mathbb{R}_{+}$is lower self-controlled (at the origin) if any of the following conditions holds.

- $\varphi$ is radially non-decreasing from the origin.

- $\varphi$ is Lipschitz, i.e. as in (3).

- $\mathbb{H}^{d-1} \backslash E_{\varphi}$ is a Nagel-Stein domain.

Proof. The first statement is trivial.

The second is also easy. Suppose that $|\varphi(x)-\varphi(y)| \leq a|x-y|$ holds and choose $\Delta<\frac{1}{a}$, see Definition 2.2. Furthermore, suppose that $y=x+v, \operatorname{sign}\left(v_{i}\right) \operatorname{sign}\left(x_{i}\right) \geq 0$ and $|v|<\varphi(x) \Delta$. Then

$$
\varphi(x)-\varphi(y) \leq|\varphi(x)-\varphi(y)| \leq a|v| \leq a \Delta \varphi(x)
$$

due to the assumptions.

Thus, we have that

$$
\frac{\varphi(x+v)}{\varphi(x)} \geq 1-a \Delta>0 .
$$

The last statement follows easily from the definition of a Nagel-Stein domain, see for example [3].

Remark 3.5. Note that there are no implications between the first two conditions in the above lemma. Note also that the third condition implies the second.

3.1. A lower self-controlled example. Let $u$ and $v$ be two Lipschitz functions from $\mathbb{R}$ to $\mathbb{R}_{+}$and such that $u(0)=v(0)=0, u(x)>v(x)$ when $x \neq 0$, and such that $E_{u}$ is not minimally thin at 0 , but $E_{v}$ is minimally thin at 0 , in the upper-half-plane.

Let us now construct a new function, $w$, that "zig-zags" its way between $u$ and $v$ in the following way: Let $\lim _{x \nearrow-1} w(x)=u(1)>v(1)=\lim _{x \searrow-1} w(x)$, and whenever the right limit of $w$ equals $v, w$ will increase linearly with a slope $k$ if $x<0$. See Figure 2. A similar construction is made for $x>0$. The resulting function is neither Lipschitz nor monotone, but it will be lower self-controlled. Hence one can use the integral criterion (4) for $w$ to decide whether $E_{w}$ is minimally thin at 0 .

\section{REFERENCES}

[1] H. Aikawa On the behavior at infinity of non-negative superharmonic functions in a half space. Hiroshima Math. J. 11 (1981), 425-441.

[2] H. Aikawa Quasiadditivity of Riesz capacity, Math. Scand. 69 (1991), 15-30.

[3] H. Aikawa Thin sets at the boundary, Proc. London Math. Soc. (3) 65 (1992), 357-382.

[4] A. Beurling A minimum principle for positive harmonic functions, Ann. Acad. Sc. Fenn. $372,(1965), 3-7$. 


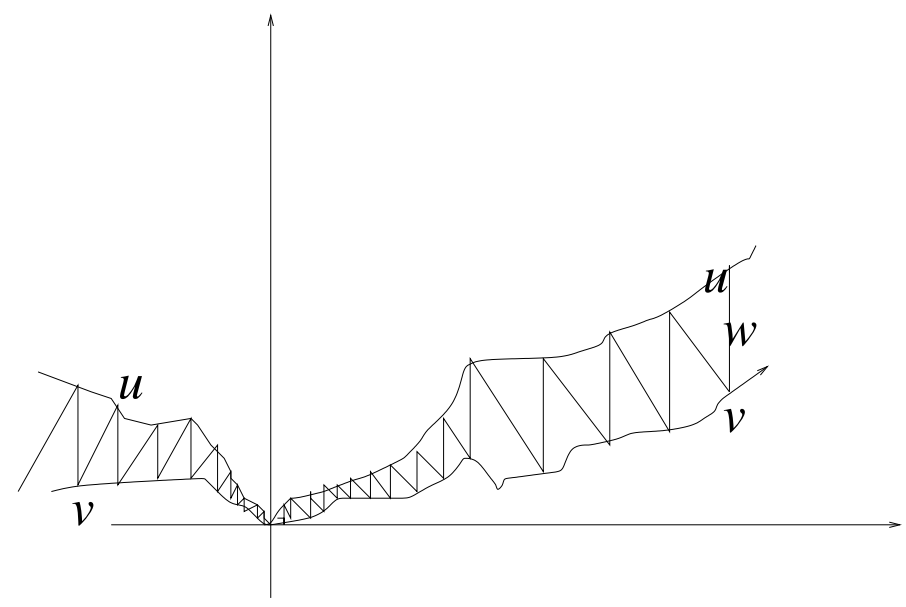

Figure 2. A lower self-controlled zig-zag function which is neither monotone, nor Lipschitz.

[5] N. H. Bingham, C. M. Goldie and J. L. Teugels Regular variation, Cambridge University Press, Cambridge (1987).

[6] M. Brelot and J.L. Doob, Limites angulaires et limites fines, Ann. Inst. Fourier, Grenoble 13 (1963), 395-415.

[7] K. Burdzy Multidimensional Brownian excursions and potential theory, Pitman Research Notes in Mathematics Series 164, Longman Scientific \& Technical (1987).

[8] B. Dahlberg A minimum principle for positive harmonic functions, Proc. London Math. Soc. (3) 33 (1976), 238-250.

[9] M. Essén On minimal thinness, boundary behavior of positive harmonic functions and quasiadditivity of capacity, Proc. Edinburgh Math. Soc. 36 (1992), 87-106.

[10] S. J. Gardiner A short proof of Burdzy's theorem on the angular derivative, Bull. London Math. Soc. 23 (1991), 575-579.

[11] C. M. Goldie and R. L. Smith Slow variation with remainder: theory and applications, Quart. J. Math. Oxford (2), 38 (1987), 45-71.

Mathematical Sciences, SE-412 96 Göteborg, Sweden

E-mail address: torbjrn@math.chalmers.se 